\title{
SPRECHSTUNDE NATURHEILKUNDE
}

\section{Polyneuropathien}

Patienten mit polyneuropathischen Beschwerden sind durch Schmerzen und Bewegungseinschränkungen in ihrer Lebensqualität stark beeinträchtigt. Die verfügbare medikamentöse Standardtherapie ist in ihren Möglichkeiten begrenzt und geht oft mit nicht unerheblichen Nebenwirkungen einher. Naturheilverfahren können - ergänzend eingesetzt - die Behandlungserfolge erhöhen.

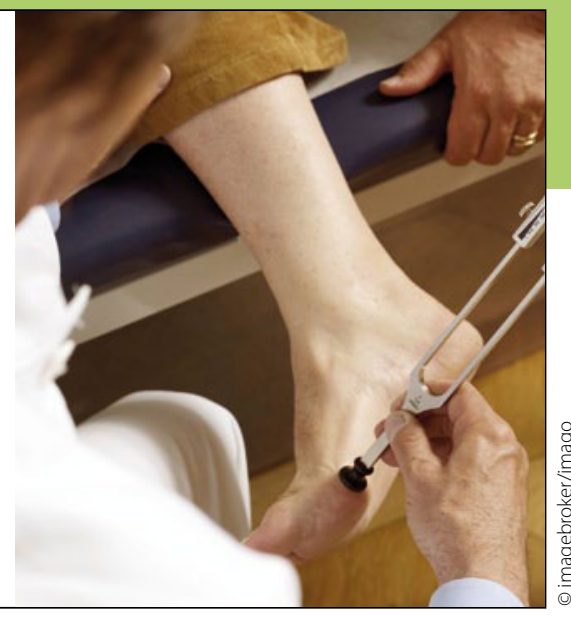

- Besonders bei idiopathischen Formen der Polyneuropathie ist die Behandlung schwierig und das Spektrum der therapeutischen Möglichkeiten begrenzt. Im Folgenden werden die wichtigsten naturheilkundlichen Verfahren vorgestellt, durch die die Behandlung ergänzt werden kann.

\section{Hydrotherapie}

Ansteigende Armbäder verbessern konsensuell die periphere Durchblutung und führen zu einer reflektorischen $\mathrm{Ge}$ fäßerweiterung auch der unteren Extremitäten.

Für die Durchführung gelten folgende Empfehlungen: Der Patient sollte die Armbäder im Sitzen vornüber gebeugt mit verschränkten Armen im Waschbecken durchführen. Man beginnt mit neutralen Badetemperaturen um $35^{\circ} \mathrm{C}$ und lässt langsam heißes Wasser zufließen, sodass nach drei bis fünf Minuten ca. $40^{\circ} \mathrm{C}$ erreicht werden. (Temperaturkontrolle durch Thermometer!)

$\mathrm{Zu}$ Hause sollte der Patient regelmäßig wechselwarme Kneippsche Güsse durchführen. Umschläge mit Heilerde an den Unterschenkeln und Füßen haben einen antiphlogistischen und schmerzlindernden Effekt.

Zur Verbesserung der Durchblutung der unteren Extremitäten hat sich auch die Schröpftherapie im Sakralbereich bewährt.

\section{Physiotherapie}

Zur Erhaltung der Mobilität steht bei Gangstörungen und schwacher Muskulatur die Bewegungstherapie im Vordergrund. Physiotherapeutisch kommen die propriozeptive neuromuskuläre $\mathrm{Fa}$ zilitationstherapie (PNF) oder auf dem Bobath-Konzept basierende Übungen zum Einsatz. Ziel ist es, die Insuffizienz der Rumpfmuskulatur zu beheben und die Körperhaltung für den Alltag zu schulen.

\section{Ernährungstherapie}

Eine vollwertige ausgewogene Kost mit einem hohen Anteil an Obst und Gemüse ist wichtig, um eine ausreichende Vitaminzufuhr zu gewährleisten. Ggf. ist die Substitution von B-Vitaminen, Folsäure und Eisen nach vorheriger Laborkontrolle angebracht. Häufig liegt ein Grundproblem der Polyneuropathie in der meist jahrelang bestehenden Fehlund Überernährung wie z. B. beim Diabetes mellitus. Basiert die Polyneuropathie auf einem Diabetes, steht die Ernährungstherapie an erster Stelle, da sich erfahrungsgemäß bei Besserung des Blutzuckerstoffwechsels auch die polyneuropathischen Beschwerden bessern.

\section{Phytotherapie}

Eine adjuvante Therapie mit Johanniskraut als Monopräparat oder ggf. in Kombination mit Baldrianwurzel und Passionsblumenkraut kann bei gleich- zeitig bestehender Dystymie angebracht sein. Salben auf Cayennepfefferbasis (Capsaicin), dünn(!) mehrmals täglich aufgetragen, führen nach anfänglichem Brennschmerz zu einer länger anhaltenden Schmerzlinderung. Extrakte aus Eisenhut (Aconitum napellus) sind als Urtinktur zu toxisch, haben sich jedoch in homöopathisch niedrig potenzierter Form als äußere Öleinreibung bewährt. Auch mit Extrakten aus Teufelskralle, Weidenrinde oder, bei akuten Schmerzattacken, mit einer Mischung aus Eschenrinde, Zitterpappelrinde und -blättern und Echtem Goldrutenkraut lassen sich die Schmerzen günstig beeinflussen.

\section{Ordnungstherapie}

Als Bindeglied zwischen der somatischen und der psychosomatischen $\mathrm{Me}$ dizin hilft die Ordnungstherapie den Patienten, besser mit ihren Beschwerden umzugehen. Gerade bei metabolischer Stoffwechselstörung mit der Polyneuropathie als Sekundärschaden ist die Stärkung der Eigenverantwortung wichtig, um längerfristig z.B. die erforderliche Ernährungsumstellung und bessere Blutzuckereinstellung durchzuhalten.

Insgesamt muss das naturheilkundliche Therapiekonzept der jeweiligen Ursache der Polyneuropathie (diabetisch, alkoholisch etc.) angepasst werden.

Dr. med. St. Fey, Prof. Dr. med. A.-M. Beer, Hattingen 